Volume 8, No.1.6, 2019

International Journal of Advanced Trends in Computer Science and Engineering

Available Online at http://www.warse.org/IJATCSE/static/pdf/file/ijatcse1181.62019.pdf

https://doi.org/10.30534/ijatcse/2019/1181.62019

\title{
A Social Media Services Analysis
}

\author{
Ummu Hani' Hair Zaki ${ }^{1}$, Roliana Ibrahim ${ }^{2}$, Shaliza Abd Halim ${ }^{3}$ \\ ${ }^{1}$ Department of computer Science, ${ }^{2}$ Department of Information Systems, ${ }^{3}$ Department of Software Engineering, \\ School of Computing, Faculty of Engineering, Universiti Teknologi, Malaysia (UTM), Malaysia
}

\section{ABSTRACT}

The growing adoption of social media services such as YouTube, Facebook, and Twitter have created opportunities for information dissemination practise that has not existed before. This practice improves situational awareness and eases dissemination of information. However, the major challenge is to efficiently extract relevant information from the large volumes of noises. It makes the social data analysis task is extremely labour intensive and time-consuming. To overcome these challenges, we propose a service-based approach to illustrate and analyse social media services. First, we assign social media as a service, called social media service. We also identify its functional and nonfunctional features. Secondly, we plan experiments on realworld datasets for a variety of topics. We analytically evaluate the functional and non-functional features of social media services. The analysis has depicted that classifying functional features and identifying non-functional features such as relatedness, preferences, and engagement of social media services is important regarding analysing social sensors data. This work demonstrates the competence of social media services regarding the Social Media ServiceBased Analysis Model. The identified functional and nonfunctional features in this work help data analyst to understand and comprehend the nature of social media services particularly in the subject of Syria war, dengue outbreak and natural disaster. Furthermore, we have provided future research issues from where our work has ended.

Key words : Social media, Social media service, Social sensor data, Functional features, Non-functional features.

\section{INTRODUCTION}

The prominent social media like Facebook, Twitter, YouTube, and Instagram have appeared to be a source of free data. Due to that, users speedily create and post the data using social media services. Sakaki et al., [1] introduced social media users as human sensors or social sensors. Study that benefiting various social media data included research fields such as natural disaster detection [2], public health surveillance [3], hate crime sentiment [4], Arabic language sentiment [5], mobile device brand evaluation [6], and social media influencer [7]. According to the "New 2018 Global Digital", a suite of reports from "wearesocial.com" and "hootsuite.com", the number of social sensors worldwide in 2018 is 3.196 billion. Social sensor obtains and share information instantly on a $24 / 7$ basis and provided an overview of the situation at hand [8]. Based on Mondovo", Facebook continues to hold tight to its title as the number one most popular social media platform out there with every day, 35 million people update their statuses on Facebook. It followed by YouTube as the second most searched keyword on Google and having over 4 billion videos viewed in a day [9]. While Twitter is famously analysed more in academic publications [10]-[12], Hootsuite ${ }^{2}$ reported that it reached until 500 million tweets sent each day. The multiple purposes for these three social media and its international and inter-generational audiences make it a potentially valuable source of information about the issues depicted in them [13].

Although these platforms are primarily used for online data sharing, social media data possess unique characteristics. They differ in terms of social sensor participation and sharing mechanisms [14]. For example, Twitter is textbased social media where the users only get 280 characters per tweet. YouTube is a video-based social media where users publish videos on any topics. Viewers may respond to their opinions by commenting on the content in a text form. In addition, various topics may influence data features such as the sheer volume of content and data quality. For example, every minute on Facebook ${ }^{3}$, 510,000 comments are posted, 293,000 statuses are updated, and 136,000 photos are uploaded. There are a lot of engaged and active users, but also a huge amount of information competing for their attention, so quality and strategy on your part matter. And this is only one of the available social media platforms. There is clearly too much data to analyse. Such characteristics affect the quality of social media service [15]-[17]. Despite that, available social media analysis tools treat social media platforms as a uniform data source with similar properties [18]. That means available tools lack the flexibility to differentiate between social media platforms based on their diverse features. In this work, we are addressing how social sensor data produced by different social media services can be used to provide better situational awareness. Therefore, this paper suggests that to

\footnotetext{
${ }^{1}$ https://goo.gl/NmZzCE

${ }^{2}$ https://goo.gl/rTpxBc

${ }^{3}$ https://bit.ly/2jNR9SO
} 
effectively analyse social media data, it is important to study the diversity of social media platforms and its unique characteristics.

There are several types of research that analyse the features of social media platforms. Dai et al. [14] identified four quality dimension of social media in the domain of Knowledge Management (KM) which are system usability, content exchangeability and accessibility, content quality and, sociability. The mean scores of content accessibility and content quality dimension are the highest, that indicated that "content" is appeared to be central to the social media platforms. Users prefered to such platforms for good content generated [19]. Andrews et al. [20] assessed the content of Twitter data regarding Colorado wildfires with two quality dimension which is credibility and informativeness. The quality dimension is classified based on two scopes such as tweets and message-related (length of the text of the tweet (in characters); the number of words; a fraction of capital letters in the tweet; the number of URLs contained in a tweet; username mentions; hashtag).

Neppalli et al. [21] studied how the divergence of sentiments in a tweet posted during Hurricane Sandy 2012 affects the tweet retweet-ability. They analysed the impact of Emotional Divergence (ED) on the retweet-ability of tweets during Hurricane Sandy. ED in a given short text measures the spectrum of the emotions expressed in it, whereas emotional polarity (sentiment) capture the overall emotion from the text. During natural disasters, identifying how likely a tweet is to be retweeted is important since it can help promote the spread of "good" information on Twitter, as well as it can help stop the spread of misinformation. Another interesting pattern that they discovered is that the content of tweets with low ED is informative in nature, whereas the content of tweets with high ED is more of individual opinions and do not necessarily convey any useful information.

These works defined how social media platforms are supposed to be. Apparently, they addressed the nonfunctional features of social media platforms, simply, the quality features. As a different perspective from the aforementioned studies, our goal in this research study pays specific attention to the assessment and classification of functional and non-functional features of social media platforms and its unique characteristics. This paper proposes an approach for identifying and analysing social media as services. Services are autonomous and platform-independent entities. They accomplish various functions such as replying straightforward requests for completing advanced business processes. It involves peer-to-peer affairs amongst several layers of service consumers and providers. Any piece of code and any application component programmed on a system can be re-processed and changed into a network-available service.

Services involve of functional features and non-functional features. Functional features describe the task or role presented by a service whereas non-functional features define aspects such as robustness, cost, throughput, reliability, response time, and availability related to the service. However, a desired functional and non-functional requirement may not be met by a single web service [22]. Furthermore, social media as a service has an enormous number of users, the velocity data production is fast and the diversity of social sensor data in term of noise promotes research questions such as (RQ 1) what are the features of social media services in term of functional and nonfunctional perspective? (RQ 2) how particular dataset influence the functional and non-functional features of social media services?

The aim of this paper is to summarize the functional and non-functional features of social media service. This work is important in comprehending the condition and process flow of analysing social media services. This work contributes to the following part, we propose a service-based approach to illustrate and analyse social media services. Then we identify its functional and non-functional features. After that, we plan experimentations on the real-world datasets for a variety of the dataset such as natural disaster, outbreak, and war. We then analyse and assess the outcomes constructed on several functional and non-functional features. The rest of this paper is arranged as follow. Section II outlines the related works. Section III represents the analysis methodology. Section IV defines the particulars of discoveries through experimentations results. Section V specifies the detailed discussion and Section VI summarize the paper.

\section{RESEARCH METHOD}

Combining multiple services for social media are heavily constrained by its dependable workflow. The workflow must undergo thorough and proper data mining step, each step involves several functions and these functions comprise with other sub-functions. This increase the urgency to clarify $\mathbf{R Q}$ $\mathbf{1}$ and RQ 2. We plan a methodology and perform experimentation onto the collected data. For RQ 1, firstly, we obtained social sensor data from Facebook, Twitter, and YouTube. The significant of these three social media services already discussed in Section 1, Introduction. Initially, we define the service model to represent social media services. The service model is defined based on a functional model and a non-functional model. To define the service model, we first organize functional features of social media services and establish the functions for each type of social media service based on the data produced. Plus, we also suggest a non-functional model that selects the nonfunctional features of social media services. Meanwhile, in answering RQ 2, we assume that the non-functional features of social media services may perform uniquely for the different dataset. To test our assumption, we analyse the data from three social media services for three different datasets such as war, outbreak and natural disaster. 


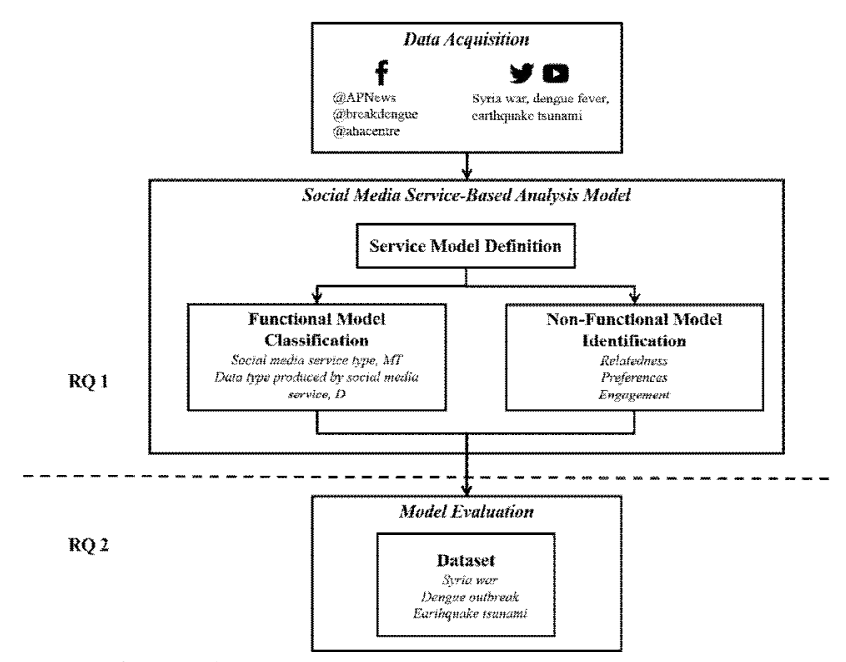

Figure 1:. Social media services-oriented analysis framework

\subsection{Data Acquisition}

We obtained the social sensor data from three types of social media services, for instance, Facebook, Twitter, and YouTube. The data is acquired using Facepager [23]. Facepager made for fetching publicly available data from Facebook, Twitter, and other JSON-based APIs. Facebook provides access to the data hosted on it; however, to access, is much more limited. Therefore, we propose to utilize content posted directly to the relevant pages extracting both posts and comments. To collect Facebook data such as status posts or comments from the Facebook page, Facepager is using "Graph API" service. For Twitter, Facepager collects tweets using "Twitter Streaming API" service. While for YouTube, we are also using Facepager that extracting video title and its comment count. We have three subjects for dataset such as war, outbreak, and natural disaster as tabulated in Table 1. The data is collected for 11 days from 04 March 2019 to 14 March 2019. We are also doing some advanced searching during data acquisition such as language filtration, where we only select data constructed in the English language. In the YouTube environment, there are 3 resources such as channel, playlist, and video. We only use video resource. Besides, we select several keywords during the data acquisition process as shown in Table 1.

Table 1: Dataset collected by keyword selection

\begin{tabular}{lll}
\hline Dataset & Facebook & Twitter YouTube \\
\hline War $(W)$ & @APNews & $\begin{array}{l}\text { Syria war, dengue } \\
\text { fever, earthquake- } \\
\text { tsunami }\end{array}$ \\
Outbreak $(O)$ & $@$ breakdengue & \\
Natural Disaster $(N D)$ & @ahacentre &
\end{tabular}

\subsection{Service Model Definition}

In this subsection, we layout the explanation for RQ $\mathbf{1}$ where we are designing a service model that is established with related to the functional model, and non-functional model. The design of the service model is constructed as a tuple of five variables: $\langle I D, M, M T, D, N F\rangle$. We defined the variables in the form of simple sets notation to easy understand, where:

$$
\begin{aligned}
& I D=\{\text { service id }\} \\
& =\left\{\text { service }_{1}, \text { service }_{2}, \text { service }_{3}, \ldots, \text { service }_{n}\right\} \\
& M=\{\text { set of actual social media services }\} \\
& =\{\text { Facebook, Twitter, YouTube, etc }\} \\
& M T=\{\text { type of social media services }\} \\
& =\{\text { social networking, microblogging, multimedia } \\
& \text { sharing }\} \\
& D=\{\text { data type produced by social media services }\} \\
& =\{\text { text, response, } \text { multimedia }\} \\
& N F=\{\text { set of non-functional features of social media } \\
& \text { services\} } \\
& =\left\{n f_{1}, n f_{2}, n f_{3} \ldots n f_{n}\right\}
\end{aligned}
$$

\subsubsection{Functional Model Classification}

The functional model consists of two variables of social media service which are social media service type $M T$ and data type produced by social media service $D$. The social media service type $M T$ described its common functions while the data type produced by social media service $D$ represents the data created. We classified the $M T$ entities in the service model such as listed below.

- Social networking $S N$. These are used to communicate informally or professionally with others, find people and share similar interests. It allows another social sensor with access to the network to directly connect with one another through groups, pages, and location. The social sensor enables to react with share images, videos or even text message and status.

- Microblogging $\boldsymbol{M B}$. It is an online broadcast medium that enables posting of short text entries or updates. The social sensor can create and share hashtags to share content about related subjects.

- Multimedia sharing $M S$. It permits the social sensor to publish videos, audio, and photos. It enables them to share publicly or privately. The social sensor may decide to add text content like captions, mentions of other users, or filters that make you look better. Another social sensor could respond with comments, dislikes or likes.

Data type produced by social media service, $D$ can be classified into three types such as text, response, and multimedia. Text data type stores any type of text data that hold integer, letters, and special characters. Response data type consists of interaction in term of like, share, and retweet. While multimedia data type in this work includes images, graphics objects, audio, video, animation sequences. In this work, we only analysed functional features focus on text and response data type. But for classification purpose, the multimedia data type is listed. 
Ummu Hani et al., International Journal of Advanced Trends in Computer Science and Engineering, 8(1.6), 2019, 69 - 75

Table 2: Functional features classification

\begin{tabular}{|c|c|}
\hline $\mathbf{M}$ & MT D \\
\hline Facebook, LinkedIn, or Google+ & SN Text, response, multimedia \\
\hline Twitter or Tumblr & MB Text, response \\
\hline Instagram, YouTube, or Snapchat & MS Response, multimedia \\
\hline
\end{tabular}

\subsubsection{Non-Functional Model Identification}

We determine the non-functional variables $N F$ based on datatype produced by social media service $D$. The nonfunctional features that have been used are relatedness, preferences, and engagement. Relatedness means that the data collected is related to the dataset. Preparing texts for analysis requires making choices that can affect the accuracy, validity, and findings of a text analysis study as much as the techniques used for the analysis [24]. While preferences defined the social sensor willingness to share their data or to refer to particular social media service despite another alternative social media service. Besides, engagement described social sensor data contains the Interactions $I$ indicates what is being done and the Conversations $C$ demonstrates what is being said [25]. Social sensor engages with one another by interacting with likes, share, retweet, repost and by conversation through reply and comments. The number of interaction and conversation indicates a high engagement rate.

\section{RESULTS AND ANALYSIS}

In this section, we discussed RQ 2. We analyse the functional and non-functional features of our dataset regarding three datasets and demonstrate the analysis results.

\subsection{Functional Features Analysis}

The functional model that has been classified in Section 2.2.1 shows that social media services produce a particular data type. Each of them has a unique niche for different dataset. For example, the critical subject such as war or natural disaster, Twitter produce a justifiable amount of data. Social sensor quickly updates situational information compare to Facebook and YouTube, not denying the convenience in using both of it. The functional features of social media services are analysed referring to the three datasets which are war, outbreak, and natural disaster. Table 2 shows the analysis results. Based on Table 2 from functional model classification, we are choosing one social media for every type of social media service. The dataset is collected for 11 days. Data type produced by social media service $D$ consists of comments from a Facebook post, tweets, and comment thread from YouTube video. Based on Table 2, it shows that every social media service has a distinct imbalance in the total dataset for every subject. Twitter shows the highest amount of dataset collected due to its service which is "Twitter Streaming API" service. Social sensors composed a small amount of data on Facebook for dengue outbreak subject.
As a functional model consists of two variables of social media service $M T$ and $D, M T_{\text {Twitter }}$ generated the most amount of data due to the short data sharing limit (280 characters). $M T_{\text {Twitter }}$ got a faster rate of new information exchange. Although the social sensor can immediately share things on Facebook, Twitter originated the retweet $(R T)$. This will automatically get the news out to whoever follows the people that $R T$ their post. Besides, $M T_{\text {Twitter }}$ allows a social sensor to accumulate an unlimited number of followers and to broadcast to them with zero engagement or interactivity if they choose to do so. At the beginning of 2018, the Facebook algorithm change ${ }^{4}$ that shows the platform's users more of what they want and less of what they do not. The other aim was to keep users on the platform longer. It also meant, marketing content was poised to take a backseat to the content by friends and family. As the dataset is collected from the Facebook page, comments from Facebook page fans or even other users is lesser compared to $M T_{T_{w i t t e r}}$ and $M T_{\text {Youtube }}$.

Table 2: Functional Features Analysis by Dataset

\begin{tabular}{|c|c|c|c|c|c|c|c|c|c|}
\hline \multirow{4}{*}{$\begin{array}{l}\text { Functional } \\
\text { Dataset }\end{array}$} & \multicolumn{9}{|c|}{ Type of social media service $M T$} \\
\hline & \multirow{2}{*}{\multicolumn{3}{|c|}{$\begin{array}{c}\text { SN Facebook } \\
\begin{array}{c}\text { Post/comment } \\
\text { s }\end{array} \\
\end{array}$}} & \multirow{2}{*}{\multicolumn{3}{|c|}{$\begin{array}{c}M B \text { Twitter } \\
\text { tweets }\end{array}$}} & \multirow{2}{*}{\multicolumn{3}{|c|}{$\begin{array}{c}\text { MS YouTube } \\
\text { comments }\end{array}$}} \\
\hline & & & & & & & & & \\
\hline & $\mathbf{W}$ & $\mathbf{O}$ & ND & $\mathbf{W}$ & $\mathbf{O}$ & ND & $\mathbf{W}$ & $\mathbf{O}$ & ND \\
\hline 04.03 .19 & 21 & 2 & 1 & 2,334 & 104 & 65 & $\begin{array}{c}1 \mathrm{Vid} / \\
162\end{array}$ & 0 & 0 \\
\hline 05.03 .19 & 34 & 2 & 0 & 2,552 & 105 & 129 & $\begin{array}{c}1 \mathrm{Vid} / \\
463\end{array}$ & 0 & 0 \\
\hline 06.03 .19 & 25 & 2 & 2 & 3,891 & 166 & 266 & $\begin{array}{l}1 \mathrm{Vid} / \\
3454\end{array}$ & 0 & $\begin{array}{l}2 \mathrm{Vid} / \\
2819\end{array}$ \\
\hline 07.03 .19 & 25 & 1 & 1 & 3,881 & 220 & 184 & $\begin{array}{c}1 \mathrm{Vid} / \\
127\end{array}$ & 0 & 0 \\
\hline 08.03 .19 & 33 & 2 & 3 & 2,730 & 228 & 417 & $\begin{array}{c}1 \mathrm{Vid} / \\
738\end{array}$ & 0 & $\begin{array}{c}4 \mathrm{Vid} / \\
1760\end{array}$ \\
\hline 09.03 .19 & 17 & 0 & 2 & 2,917 & 80 & 176 & $\begin{array}{c}8 \mathrm{Vid} / \\
741\end{array}$ & $\begin{array}{c}2 \mathrm{Vid} / \\
319\end{array}$ & $\begin{array}{c}2 \mathrm{Vid} / \\
26\end{array}$ \\
\hline 10.03 .19 & 17 & 0 & 0 & 3,148 & 54 & 648 & $\begin{array}{c}8 \mathrm{Vid} / \\
185\end{array}$ & $\begin{array}{l}8 \mathrm{Vid} / \\
259\end{array}$ & $\begin{array}{c}3 \mathrm{Vid} / \\
16\end{array}$ \\
\hline 11.03 .19 & 38 & 2 & 0 & 5,571 & 73 & $\begin{array}{c}6,07 \\
7\end{array}$ & $\begin{array}{c}8 \mathrm{Vid} / \\
811\end{array}$ & $\begin{array}{c}11 \mathrm{Vid} / \\
360\end{array}$ & $\begin{array}{c}11 \mathrm{Vid} / \\
559\end{array}$ \\
\hline 12.03 .19 & 25 & 1 & 0 & 5,093 & 105 & $\begin{array}{c}1,31 \\
1\end{array}$ & $\begin{array}{l}8 \mathrm{Vid} / \\
244\end{array}$ & $\begin{array}{c}8 \mathrm{Vid} / \\
146\end{array}$ & $\begin{array}{c}\text { 6Vid / } \\
639\end{array}$ \\
\hline 13.03 .19 & 12 & 2 & 1 & 4,125 & 106 & 382 & $\begin{array}{c}10 \mathrm{Vid} / \\
878\end{array}$ & $\begin{array}{c}5 \mathrm{Vid} / \\
12\end{array}$ & $\begin{array}{c}7 \mathrm{Vid} / \\
1767\end{array}$ \\
\hline 14.03 .19 & 11 & 1 & 1 & 727 & 24 & 82 & $\begin{array}{c}10 \mathrm{Vid} / \\
569\end{array}$ & $\begin{array}{c}6 \mathrm{Vid} / \\
128 \\
\end{array}$ & $\begin{array}{c}6 \mathrm{Vid} / \\
303\end{array}$ \\
\hline VOLUME & 258 & 15 & 11 & $\begin{array}{c}36,96 \\
9 \\
\end{array}$ & $\begin{array}{c}1,26 \\
5 \\
\end{array}$ & $\begin{array}{c}9,73 \\
7 \\
\end{array}$ & $\begin{array}{c}57 \mathrm{Vid} / \\
8,372 \\
\end{array}$ & $\begin{array}{c}40 \mathrm{Vid} / \\
1,224 \\
\end{array}$ & $\begin{array}{c}41 \mathrm{Vid} / \\
7,889 \\
\end{array}$ \\
\hline AVERAGE & 94. & $67 \approx$ & & 15,990 & $33 \approx 1$ & 5,990 & 5,82 & $28.33 \approx 5$, & ,828 \\
\hline
\end{tabular}

\subsection{Non-Functional Features Analysis}

For non-functional model, we are analysing three features such as relatedness, preferences, and engagement according to the dataset. For each of the proposed non-functional features, we used several techniques on handling the

\footnotetext{
${ }^{4}$ https://bit.ly/2IbDC54
} 
experiments. To clean the unrelated data from the dataset, again, we are using an $\mathrm{R}$ programming language with the below filtering mechanism. First, we are excluding English stop word and words that usually are not related to the context of dataset. For example, a music band, The Gluts, release new album 'Dengue Fever Hypnotic Trip' on April 12, 2019. The data contain the keyword dengue fever. However, it is not related to the context of dataset. Therefore, 'Hypnotic Trip' is selected as a stop word to be removed from the dataset. Secondly, we eliminated the noise such as punctuation characters, emoticons, hashtags, @ mention, and RT term. Then, we are included the related data based on significant keyword for example "Bashar alAssad" and "Aleppo" are used to be selected for war subject. Extracting essential information from social media service is not an easy task. As we are collecting data from the official page on Facebook, the nature of data is structured and tolerable to understand. This is because this page representing their organisation thus, they are thoughtful in posting their update. However, the nature in YouTube and Twitter that consider being casual and informal, the text style is ignored most of the time. due to the data type produced from different social media services, efficient noise filtering techniques is one of the issues need to be treated.

In our analysis, we found that many social sensors are a heavy user of YouTube, especially on natural disaster subject. Figure 1, Figure 2, Figure 3 shows the power of social media service that attracts social sensors to reach them out. As for the preferences, we are measuring the choice made by the social sensor to connect themselves with other regards to the dataset. We are considering "statistics.commentCount" parameter to measure YouTube user preferences. Twitter dominates the social media landscape when it comes to war dataset by considering "screen_name" count that reflect Twitter user are retweeting or tweeting about war particularly, Syria war. While Facebook is measured by "talking_about_count" parameter. Every social media service is unique, and it depends on data analyst to comprehend what resonates better with social sensors.

We define engagement features into two types: Interactions $I$ (what is been done) and Conversations $C$ (what is being said). Interaction describes the reaction, retweet, favourite, dislike, share, view, etc. while conversations refer to comments and replies. The higher the interaction and conversations rate, the better a social sensor or an organisation of being engaged, connected, involved, influenced, and interested in the dataset. Thus, prove that the dataset needs wider public coverage in that particular social media service and visibility to draw situational awareness. Figure 4 shows the engagement result of every social media service regarding dataset.

In the engagement feature, we are handling Facebook engagement by using "reactions.summary.total_count" in interaction type, and "comments.summary.total_count" in conversation type. While for YouTube, we are manipulating

several parameters such as "statistics.commentCount," "statistics.viewCount," "statistics.favoriteCount," "statistics.dislikeCount," and "statistics.likeCount". For Twitter, we are using "retweet_count" and "favourites_count" to measure the engagement. It shows that social sensor highly depends on Twitter when it comes to natural disaster subject. Twitter becomes the most engaged social media service. The essence of driving that engagement is nailing the length of the text message. Optimize the length of content and that social media service will be more likely to engage.

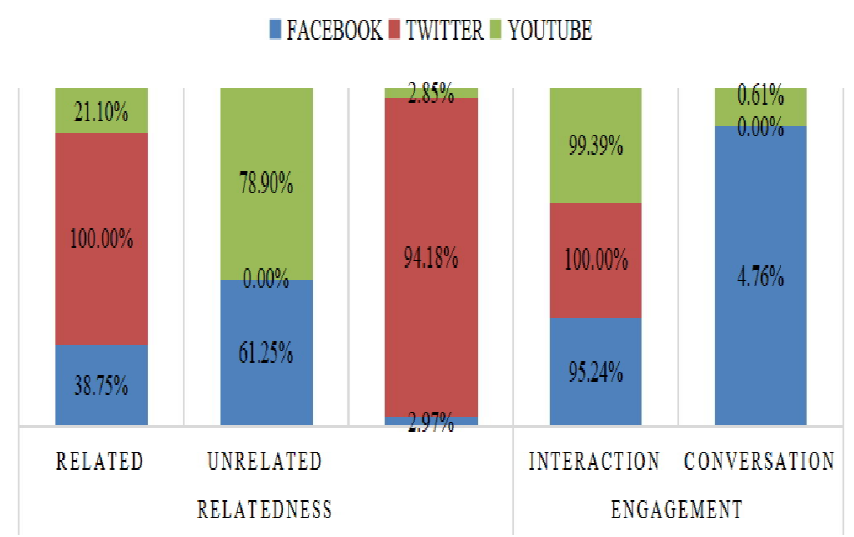

Figure 1: Syria war dataset

\section{— FACEBOOK — TWITTER — YOUTUBE}
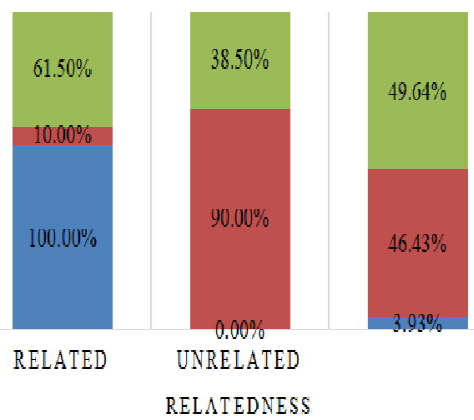

Figure 2: Dengue outbreak dataset — HACEBOOK — IWIITER — YOUIUBE

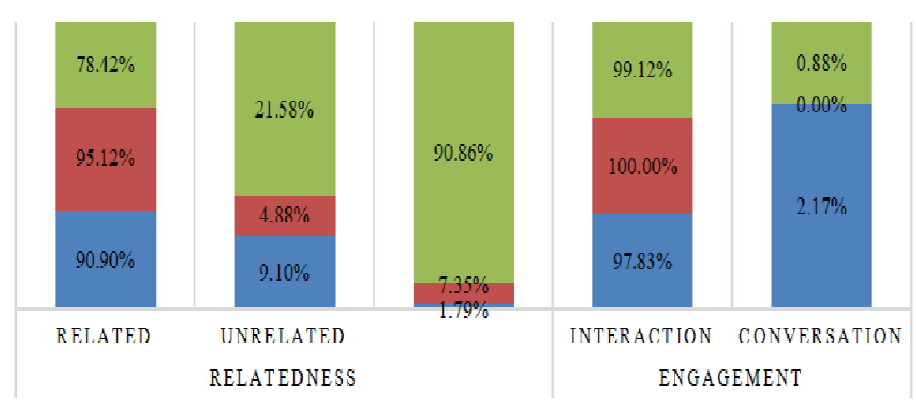

Figure 3: Earthquake tsunami dataset 
Ummu Hani et al., International Journal of Advanced Trends in Computer Science and Engineering, 8(1.6), 2019, 69 - 75

\section{CONCLUSION}

The use of social media has become widespread and can serve a variety of purposes. Social media platforms are diverse and different subject mentions affect their data features. For example, on account of situational information either during the war, disease outbreak or natural disaster event, analysing information may provide an accurate perception of the situation, ability to quickly recognize a change in that situation, understanding the impact of any change and being able to project the situation soon.

RQ 1. Thus, to efficiently analyse social media platform, we constructed social media service features using sets notation to model this analysis. Social media data possess unique characteristics that consist of functional features (a type of social media services $D$ and data type produced by social media services $M T$ ) and non-functional features (relatedness, preferences, and engagement). We found out that, the identified functional and non-functional features in this work help data analyst to understand and comprehend the nature of social media services.

RQ 2. We collected social media data from three platforms such as Twitter, Facebook, and YouTube with three different subjects which are Syria war, dengue outbreak and natural disaster. Facebook data is more formal in nature, while Twitter towards simple and short, and YouTube towards critical and detail in nature. Briefly, based on functional features, social sensor produced the most tweet $(D)$ in Twitter $(M T)$ regarding Syria war dataset. This proved, in non-functional feature context, which is preferences, where social sensor most attracted to engage and respond on Syria war subject. While referring to nonfunctional features, for relatedness, the most significant and related dataset is earthquake-tsunami dataset produced by Facebook. This is because of the nature of the Facebook page that represents an organisation, where they will publish less noise information. While for engagement feature, social sensor preferred dataset subject about earthquake tsunami in the YouTube platform. Distinctly, social sensor tends to fully utilize microblogging platform, $M B$ on a critical subject matter such as war and natural disaster.

Currently, we analysed only three non-functional features. In future work, we will be considering a geo-location feature. Location-tagged is one of the most important metadata within social sensor data. But sometimes, it does not embed. It is not effective to merely use the geotags attached to social sensor data for determining accurate geolocation. Likewise, we also considering comment thread during YouTube data collection to analyse the YouTube comment structure to get more extensive analysis result.

\section{ACKNOWLEDGEMENTS}

The authors wish to thank Universiti Teknologi Malaysia (UTM) and Ministry of Higher Education Malaysia (MOHE) for the funding of the research under the
Fundamental Research Grant Scheme (FRGS VotR.J130000.7828.4F856)

\section{REFERENCES}

[1] T. Sakaki, M. Okazaki, and Y. Matsuo, "Earthquake shakes Twitter users: real-time event detection by social sensors," in Proceedings of the 19th international conference on World wide web - WWW '10, 2010, p. 851. https://doi.org/10.1145/1772690.1772777

[2] A. Musaev, D. Wang, and C. Pu, "LITMUS: a multi-service composition system for landslide detection," in IEEE Transactions on Services Computing, 2015, vol. 8, no. 5, pp. 715-726.

[3] K. Ali, H. Dong, A. Bouguettaya, A. Erradi, and R. Hadjidj, "Sentiment analysis as a service: a social media based sentiment analysis framework," in 2017 IEEE International Conference on Web Services (ICWS), 2017, pp. 660-667.

[4] N. Zainuddin, A. Selamat, and R. Ibrahim, "Improving Twitter aspect-based sentiment analysis using hybrid approach," Springer Berlin Heidelberg, 2016, pp. 151-160.

[5] N. Al-Twairesh, H. Al-Khalifa, A. Al-Salman, and Y. Al-Ohali, "AraSenTi-Tweet: a corpus for arabic sentiment analysis of Saudi tweets," Procedia Comput. Sci., vol. 117, pp. 63-72, 2017. https://doi.org/10.1016/j.procs.2017.10.094

[6] R. K. Wong, C. H. Chi, Z. Yu, and Y. Zhao, "A system of systems service design for social media analytics," 2014 IEEE Int. Conf. Serv. Comput., pp. 789-796, 2014.

[7] S. Kumar and R. Rishi, "Data collection and analytics strategies of social networking websites," Proc. 2015 Int. Conf. Green Comput. Internet Things, ICGCIoT 2015, pp. 643-648, 2016.

[8] D. Pohl, A. Bouchachia, and H. Hellwagner, "Online indexing and clustering of social media data for emergency management," Neurocomputing, vol. 172, pp. 168-179, 2016. https://doi.org/10.1016/j.neucom.2015.01.084

[9] N. S. Chauhan, "Youtube data analysis using pyspark," Towards Data Science, 2018.

[10] A. Mukkamala and R. Beck, "Social media analytics to develop situation awareness - what can be learned from Twitter messages about Hurricane Sandy?," in PACIS 2016 Proceedings, 2016, vol. 165, no. June.

[11] M. Martínez-Rojas, M. del C. Pardo-Ferreira, and J. C. Rubio-Romero, "Twitter as a tool for the management and analysis of emergency situations: a systematic literature review," Int. J. Inf. Manage., vol. 43, no. April, pp. 196-208, 2018.

[12] X. Cao, P. Macnaughton, Z. Deng, J. Yin, X. Zhang, and J. G. Allen, "Using Twitter to better understand the spatiotemporal patterns of public sentiment: a case study in Massachusetts, USA," Int. 
Ummu Hani et al., International Journal of Advanced Trends in Computer Science and Engineering, 8(1.6), 2019, 69 - 75

J. Environ. Res. Public Health, vol. 15, no. 2, 2018. https://doi.org/10.3390/ijerph15020250

[13] M. Thelwall, "Social media analytics for YouTube comments: potential and limitations," Int. J. Soc. Res. Methodol., vol. 21, no. 3, pp. 303-316, 2018.

[14] Y. S. Dai, Q. Gao, Z. Fan, and R. Kang, "User perceived quality of online social information services: from the perspective of knowledge management," Proc. 2007 IEEE IEEM, pp. 482 486, 2007.

[15] H. Zade, K. Shah, V. Rangarajan, P. Kshirsagar, M. Imran, and K. Starbird, "From situational awareness to actionability: towards improving the utility of social media data for crisis response," in Proceedings of the ACM on Human-Computer Interaction, 2018, vol. 2.

[16] D. Murthy and A. J. Gross, "Social media processes in disasters: implications of emergent technology use," Soc. Sci. Res., vol. 63, pp. 356-370, Mar. 2017.

[17] Z. Jianqiang and G. Xiaolin, "Comparison research on text pre-processing methods on Twitter sentiment analysis," IEEE Access, vol. 5, pp. 2870-2879, 2017.

[18] S. Wan, C. Paris, and D. Georgakopoulos, "Improving Government Services Using Social Media Feedback," Soc. Media Gov. Serv., pp. 221246, 2015.

https://doi.org/10.1007/978-3-319-27237-5_11

[19] I. S. Makki and F. Alqurashi, "An adaptive model for knowledge mining in databases 'EMO_MINE' for tweets emotions classification," Int. J. Adv. Trends Comput. Sci. Eng., vol. 7, no. 3, pp. 52-60, 2018.

https://doi.org/10.30534/ijatcse/2018/04732018

[20] S. Andrews, T. Day, K. Domdouzis, L. Hirsch, R. Lefticaru, and C. Orphanides, "Analyzing crowdsourced information and social media for crisis management," in Application of Social Media in Crisis Management, B. Akhgar, A. Staniforth, and D. Waddington, Eds. Springer International Publishing, 2017, pp. 77-96.

[21] V. K. Neppalli, C. Caragea, A. Squicciarini, A. Tapia, and S. Stehle, "Sentiment analysis during Hurricane Sandy in emergency response," Int. J. Disaster Risk Reduct., vol. 21, pp. 213-222, 2017.

[22] H. Nacer, N. Djebari, H. Slimani, and D. Aissani, "A distributed authentication model for composite web services," Comput. Secur., vol. 70, pp. 144178, 2017.

https://doi.org/10.1016/j.cose.2017.05.008

[23] J. Jünger and T. Keyling, "Facepager." 2018.

[24] K. Welbers, W. Van Atteveldt, and K. Benoit, "Text analysis in R," Commun. Methods Meas., vol. 11, no. 4, pp. 245-265, 2017.

https://doi.org/10.1080/19312458.2017.1387238

[25] R. Vatrapu, R. R. A. O. Mukkamala, A. Hussain, and B. Flesch, "Social set analysis: a set theoretical approach to big data analytics," IEEE Access, vol. 4, 2016.

https://doi.org/10.1109/ACCESS.2016.2559584 